\title{
A Comparison of Pattern Recognition and Probabilistic Techniques for Capital Asset Deterioration
}

\author{
Matthew G. Karlaftis \\ Greek Air-Force Academy
}

\begin{abstract}
Capital asset management is a critical component of the operation of transit systems. In particular, much interest has been generated lately regarding the development of rolling stock deterioration models that can predict the future condition of a fleet from the corresponding deterioration curves. Based on a rolling stock inspection data set from Athens, Greece, this paper presents the development of both an ordered probit model and a predictive discriminant function that can be invaluable tools in predicting rolling stock deterioration. This combination of models provides a way in which we can get both aggregate (system level) projections on future bus conditions and disaggregate (individual bus level) projections. Both of the methodologies used recognize the ordinal nature of condition ratings and link deterioration to a set of relevant explanatory variables such as bus age, mileage, and size. The results can be easily used in a number of practical situations, such as capital asset life-cycle cost analysis, optimal timing for bus replacement, and examination of the effect of different operational strategies on bus deterioration.
\end{abstract}




\section{Introduction}

Capital asset management issues in the transit industry have attracted considerable research interest because of their wide variety of applications. Individual transit systems, for example, may be interested in identifying those factors that influence the deterioration of their capital assets (rolling stock and fixed facilities) to forecast the future condition of their fleet (possibly to examine the effectiveness of the current maintenance procedures) and make better investment decisions. State Departments of Transportation (DOTs), on the other hand, may wish to identify the present condition of capital assets (especially rolling stock), as well as forecast the percentage distribution of the condition of assets in the future at an aggregate (statewide) level. This information is essential, both for prudent capital funding requests and the completion of an effective Public Transit Management System (PTMS). Traditionally, these problems have been addressed in fairly simplistic and theoretically questionable frameworks. These models may not depict accurately the qualitative and quantitative relationships between capital stock deterioration and the various independent variables.

Capital asset condition is most often represented by inspection ratings (FTA 1994). Ratings are discrete ordinal measurements; that is, numbers assigned do not indicate distances between ratings, but only a relative ordering. For example, bus condition can be described on a scale of 0 to 4 , where 4 stands for excellent condition, and 0 stands for bad condition. These discrete ratings are used instead of continuous indices, primarily for reducing the computational complexity of the Maintenance and Rehabilitation decisionmaking process. Unfortunately, deterioration models based on these discrete ratings are more complex to develop. Using ordinary regression analysis to forecast future condition (Galbraith 1996) does not recognize the discrete nature of condition ratings (the dependent variable is not continuous), and the assumption of zero mean and constant variance are not met. The purely stochastic (curve-fitting) method for predicting deterioration also suffers from the weakness that it does not link deterioration with any explanatory variables, such as age of bus, mileage, etc. 
The increasing interest in transit capital asset management was promoted by the federal government with the requirement for a PTMS with the passage of the Intermodal Surface Transportation Efficiency Act (ISTEA) of 1991. This legislation required that the states implement a PTMS as ". . . a systematic process for providing relevant parties with the information needed to make informed decisions regarding their transit assets. . .." Even though the PTMS is not a federal requirement as of 1996, states are encouraged to pursue its development. Several states (Michigan, Minnesota, California, and Indiana) and individual transit systems (New Jersey) continue to work on the development of statewide and individual PTMSs, suggesting the need for improvement of existing empirical models for rolling stock deterioration.

This paper focuses on the development of deterioration models that can be used for condition forecasting at both the aggregate and disaggregate levels. While the methodologies that we develop are applied to a rolling stock (bus) condition data base from Athens, Greece, the same methodologies easily can be used to develop deterioration models for other capital assets such as service vehicles, fixed maintenance facilities, etc. The remainder of this paper is organized as follows. In the next section, some of the necessary background for this work is provided. Then, the data, the methodology, and the estimated models used in the study are presented. Concluding remarks are presented in the final section of the paper.

\section{Background}

As previously mentioned, much of the early work in the empirical analysis of rolling stock deterioration data was done with the use of multiple linear regression. These models suffer from several methodological limitations and practical inconsistencies. To overcome these limitations, some authors developed a variety of different models that are reasonable descriptors of the bus deterioration process.

Ludwig (1997) describes the development of a deterioration model for the New Jersey Transit PTMS (individual transit system deterioration model). This 
model predicts deterioration rates as a function of the median years to transition from one condition state to the next (for example, the median time it takes for a bus to deteriorate from condition rating 4 to condition rating 3 is two years). This model, while an improvement over previously-used models, in that it recognizes the discrete nature of the dependent variable, links deterioration rates to median years to drop condition only and does not account for any other variables of interest, such as mileage and size of bus (buses of different size might have different deterioration rates). Further, this model does not allow for the possibility of keeping a bus for more that 12 years, performing a major engine overhaul, or altering maintenance practices.

Karlaftis and Sinha (1997), using a rolling stock inspection data set from Indiana, developed an ordered probit methodology for projecting future rolling stock condition. This methodology recognizes that rolling stock condition ratings are ordinal numbers. Further, and contrary to most other methods of rolling stock condition prediction, this method links deterioration to a set of explanatory variables (age, mileage, bus size, maintenance practices, and climatic region of the transit system). Their methodology provides intuitively appealing and theoretically sound models that are useful tools in projecting future rolling stock condition at the aggregate (statewide) level. Nevertheless, while this methodology easily lends itself to aggregate forecasting (at the state or individual system level), it is not easily amenable to "what-if" analyses for individual buses. That is, we cannot easily examine what the effects of changing various strategies (maintenance, driving, etc.) are on individual buses. This information could be very useful in cases where systems are considering purchasing new vehicles or are attempting to determine alternate maintenance and driving strategies to reduce a bus's deterioration.

In this paper, a discriminant (classification) function is developed for bus deterioration prediction using a data set from the Athens, Greece, Public Transportation Corporation (OASA). This model has the advantage of being easy to use and can straightforwardly provide future condition ratings for individual buses. The model and its predictions are also compared to the ordered probit model proposed by Karlaftis and Sinha (1997). 


\section{The Data}

Athens, the capital of Greece is one of the most heavily congested cities in Europe. Its population of approximately three million accounts for about 30 percent of the entire population of Greece. In 1996, there were approximately 1.25 million registered vehicles, 950,000 of which were private automobiles. OASA, the sole provider of public transportation services (government owned and operated), plays an integral role in the mobility of citizens and in the effort to relieve congestion and improve air quality in Athens. In Table 1, some of the basic operating characteristics for OASA

Table 1

Characteristics of the OASA Bus Transit System (1994)

Total Fleet Size

1,782

Vehicles in Daily Operation

Annual Vehicle-Kilometers

$92,332,000$

Annual Passengers

$431,853,000$

Routes Executed Daily

13,932

Routes per Bus in One Shift are presented.

The data used in this study were obtained from the OASA Inventory data base and includes the entire 1,782 buses used in all the routes served (by OASA) in Athens. The data set contains inspection records for the year 1996, using the condition rating system described in Table 2 . The condition rating used was pro-

Table 2

Rolling Stock Condition Ratings

Condition

Description

$0=\mathrm{Bad} \quad$ In sufficiently poor condition that continued use presents potential problems

$1=$ Poor Requires frequent major repairs

$2=$ Fair $\quad$ Requires frequent minor repairs or infrequent major repairs

$3=$ Good Requires only nominal minor repairs

$4=$ Excellent Brand new, no major problems exist 
posed by FTA (1994) and has since been extensively used in the development of bus deterioration models (Galbraith 1996, Ludwig 1997, Karlaftis and Sinha 1997).

Examining the age of the existing vehicles yields the histogram of Figure 1. It is very interesting to note that approximately 35 percent of the buses in Athens are between 3 and 5 years old, while the remainder are between 11 and 17 years of age. It does appear that there were no significant bus purchases between 1986 and 1992, and this presents a potential problem because the existing fleet will soon be too old to efficiently serve the population of Athens. Indicative of this problem is Table 3, which shows the condition rating of the existing OASA bus fleet. It is important to note that approximately 50 percent of the fleet is in either bad or poor condition. As the literature suggests (FTA 1994), when bus ratings drop to 0 ("bad"), buses are not only unable to efficiently serve the population, but, most importantly, are unsafe and should be retired from service. Frequently, when the condition rating drops to 1 ("poor"), a strong case can be made for the purchase of new buses; the expense associated with the purchase can be offset by the savings in the high maintenance expenses associated with buses in this condition.

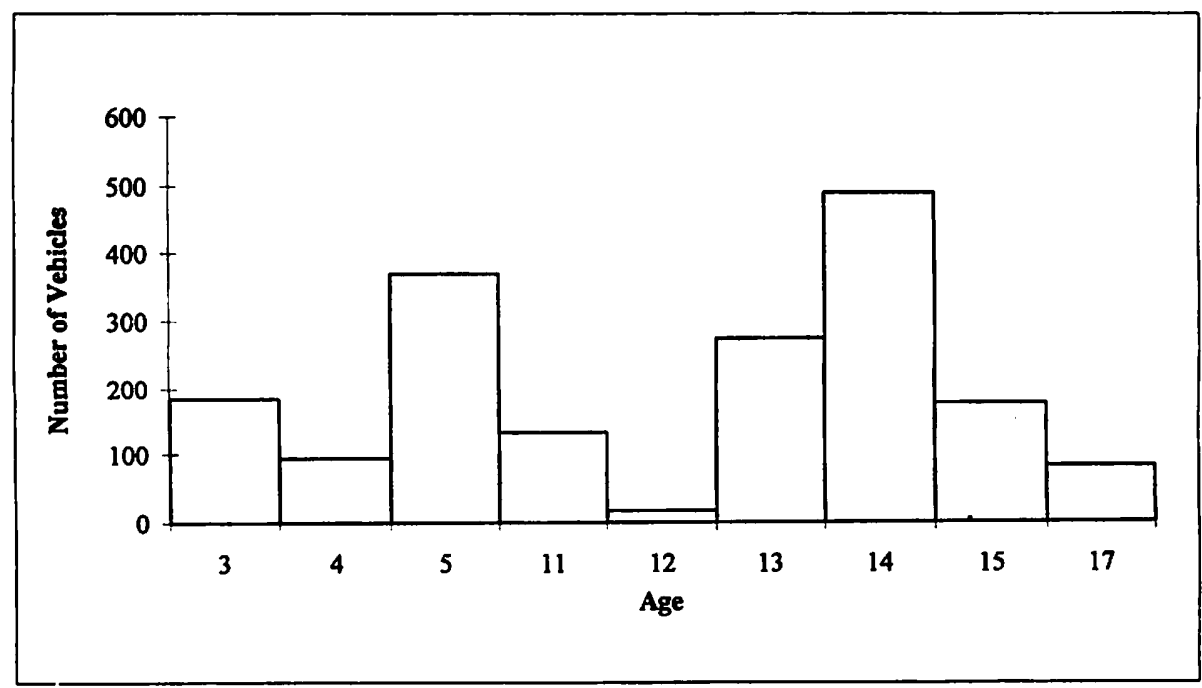

Figure 1. Age of the existing OASA bus fleet. 
To evaluate the need and timing of bus replacement, a deterioration model was developed that allows OASA to predict the condition of both its entire fleet and that of individual buses.

\section{The Methodology}

\section{The Ordered Probit Methodology}

The first model was developed primarily for aggregate forecasting and is similar to the one proposed by Karlaftis and Sinha

\begin{tabular}{|cc|}
\hline \multicolumn{2}{|c|}{$\begin{array}{c}\text { Table } 3 \\
\text { Percentage of OASA Buses in } \\
\text { Each Condition Rating }\end{array}$} \\
\hline Condition & \% of Buses \\
\hline 0 & 4.76 \\
1 & 41.38 \\
2 & 18.28 \\
3 & 20.20 \\
4 & 15.38 \\
\hline
\end{tabular}
(1997). This model is an extension of the

simple and widely used binary probit model. The ordered probit model considered here falls into the category of discrete ordered choice models. That is, this model is an extension of the probit model in which there is an ordering of the categories associated with the dependent variable (the dependent variable has a natural interpretation as an increasing integer). The ordered probit model assumes that there are cutoff points, $\mu$ 's, which define the relationship between the observed and unobserved dependent variable. ${ }^{2}$ Specifically, the ordered probit model is built around a linear-in-the-parameters latent regression, linking the latent deterioration $y^{*}$ and a set of observable exogenous variables as follows:

$$
y^{*}=\mathbf{b}^{\cdot} \mathbf{X}_{i}+\varepsilon_{i}
$$

where,

$$
\begin{array}{ll}
\mathbf{b} & =\text { a vector of parameters to be estimated } \\
\mathbf{X}_{i} & =\text { a vector of exogenous variables for bus } i \\
\varepsilon_{i} & =\text { random error term }
\end{array}
$$

The above relationship cannot be directly estimated, since $y^{*}$ is unobservable. What is actually observed is the condition ratings. These ratings, (i.e., the 
indicators of $y^{*}$ ), are used in the estimation of the deterioration model. As a result, what is actually observed in the case of bus deterioration is:

$$
\begin{aligned}
& y=0 \text { if } \quad y^{*} \leq \mu_{0}, \\
& y=1 \text { if } \mu_{0}<y^{*} \leq \mu_{1}, \\
& y=2 \text { if } \mu_{1}<y^{*} \leq \mu_{2}, \\
& y=3 \text { if } \mu_{2}<y^{*} \leq \mu_{3}, \\
& y=4 \text { if } \mu_{3}<y^{*}
\end{aligned}
$$

The $\mu$ 's are unknown parameters that are estimated along with $\mathbf{b}$. The respondents to the condition survey have their own intensity of feelings regarding the specific condition of a bus, which depends on certain measurable factors, $\mathbf{X}_{i}$ (such as mileage, age, etc.), and certain unobservable factors, $\varepsilon_{i}$. Theoretically, respondents could assign their own $y^{*}$ if they were "allowed" to do so. Given the five possible condition ratings, they choose the rating that most closely represents their own assessment of the bus condition. The parameters of the equation, as well as the thresholds and the asymptotic standard errors, are estimated simultaneously using maximum likelihood estimation.

\section{The Discriminant Analysis Methodology}

Predictive discriminant analysis (commonly referred to as classification analysis in the physical sciences or as pattern recognition in engineering and computer science) is a multivariate technique concerned with assigning objects (observations) to previously defined groups. The basic purpose of a predictive discriminant analysis (PDA) can be described as follows: ${ }^{2}$ Suppose there are samples from $\pi_{g}$ populations (condition ratings) of size $n_{g}, g=1,2, \ldots, k$, with $X$ measures (independent variables) on each of the $N\left(N=\sum n_{g}\right)$ units. Using this $N \mathrm{x} X$ data matrix, we want to determine from which of the $\pi_{g}$ populations an $(N$ $+1)$ st unit is most likely to have been randomly sampled. To accomplish this task, the maximum likelihood principle is used: Assign a unit to the population in which its observation vector has the greatest likelihood of occurrence. More formally, this can be stated as: 
$\hat{\mathrm{d}}_{\mathrm{g}}(i)=\max \left\{\hat{\mathrm{d}}_{1}(i), \hat{\mathrm{d}}_{2}(i), \ldots, \hat{\mathrm{d}}_{\mathrm{k}}(i)\right\}, i=1,2, \ldots, N, g=1,2, \ldots, k$

The functional form of the discriminant function $\mathrm{d}_{\mathrm{g}}$ depends on two factors:

1) whether the populations are normally distributed, and

2) whether the populations have equal or unequal covariance matrices.

With normal populations and equal covariance matrices, the discriminant function (DF) is linear. With normal populations but unequal covariance matrices, the discriminant function is quadratic. Finally, with non-normal populations, the discriminant function is non-linear and is estimated using nonparametric procedures (such as kernel estimation) (Huberty 1994). Selecting among these functional forms can be done by testing for normality of the populations and for equality of the covariance matrices.

\section{Model Estimation, Validation, and Forecasting}

\section{Model Estimation}

The estimation results for the ordered probit model using the OASA data set are presented in Table 4. The coefficients for the model have the expected signs: older buses, as well as buses with higher mileage (expressed as 100,000 $\mathrm{kms}$ ), are associated with lower condition ratings. Further, larger buses (higher capacity) are associated with lower condition ratings. This result seems to suggest that, in the case of OASA, either larger buses demonstrate a higher tolerance to the normal "wear-and-tear" of traffic, or that better care is taken of larger buses. It is worth mentioning that this last result (larger buses are associated with higher condition ratings) was similar to the result reported by Karlaftis and Sinha (1997).

The t-statistics for all the explanatory variables are highly significant, suggesting that all these variables are good descriptors of the bus deterioration process. The three additional parameters appearing in Table 4 (thresh 1, thresh 2, thresh 3) are the thresholds that can be statistically identified. There are four thresholds associated with five condition ratings, but the presence of a constant term in the specification of the model does not allow for the identification of one 
of the parameters. As such, the software used for the estimation of this model (LIMDEP7) normalizes the first threshold $\mu_{0}=0$. This normalization does not affect the relative values of the parameters and is done solely for estimation purposes (Greene 1990).

To estimate the second model, the DF, the normality of the populations, as well as their covariance matrices, should be checked. Investigating multivariate normality is not as straightforward as assessing univariate normality. It is very difficult to construct a test for overall test of joint normality in more than two dimensions because of the large number of things that can go wrong (Johnson and Wichern 1992). One thing that can be done is to check for the normality of each variable distribution. ${ }^{3}$ Using the SAS software (PROC

\section{Table 4}

Estimation Results for the Estimated Ordered Probit Model

\begin{tabular}{lr}
\hline Variable Name & $\begin{array}{r}\text { Ordered Probit } \\
\text { Coefficient Estimates }\end{array}$ \\
\hline Constant & 12.59 \\
& $(37.77)$ \\
Age (in years) & -0.78 \\
& $(-21.98)$ \\
Mileage (105 kms) & -1.22 \\
& $(-14.07)$ \\
Bus Capacity & 0.007 \\
& $(4.28)$ \\
thresh 1 & 2.07 \\
thresh 2 & $(35.14)$ \\
& 4.69 \\
thresh 3 & $(37.51)$ \\
Summary Statistics & 9.62 \\
\# of observations & $(42.82)$ \\
$L(0)$ & \\
$L(\beta)$ & \\
Rho-squared & 1782 \\
& -3959.21 \\
& -1049.05 \\
& 0.73 \\
& \\
& \\
&
\end{tabular}

UNIVARIATE and normal probability plots), the null hypothesis of univariate normality could not be rejected for any of the variables. Having satisfied multiple univariate normality, this investigation proceeds as though multivariate normality conditions are met. The second condition, that of equal population covariance matrices, can be examined straightforwardly since statistics are available that test this condition explicitly. The approach typically used tests the multivari- 
ate hypothesis $\sum_{1}=\sum_{2}=\ldots \sum_{k}$ (a generalization of the univariate hypothesis $\sigma_{1}$ $=\sigma_{2}^{2}=\ldots \sigma_{k}^{2}$ using an approximate chi-squared (Barlett) statistic (Huberty 1994). Using SAS DISCRIM, the reported P value of the chi-squared statistic is 0.34 , suggesting that the null hypothesis of covariance homogeneity cannot be rejected.

With normal populations and equal covariance matrices, a Linear Discriminant Function (LDF) is employed. As Lachenbruch (1975), Titterington (1981), and Gilbert (1968) suggested, LDF perform well on ordered discrete categories. The coefficients of the LDF that are estimated appear in Table 5. Notice that for each condition rating (0-4), there is a different LDF. Using these LDFs and the maximum likelihood rule, the observations to the various condition ratings can be classified.

\begin{tabular}{|lrrrrr|}
\hline \multicolumn{7}{c|}{ Table 5 } \\
& Linear Discriminant Function \\
\hline Variable & 0 & 1 & 2 & 3 & 4 \\
\hline Constant & -333.55 & -282.34 & -247.51 & -189.72 & -40.88 \\
Age (years) & 13.29 & 12.09 & 11.88 & 12.52 & 13.18 \\
Mileage (10 kms) & 42.43 & 38.42 & 35.35 & 26.27 & -12.41 \\
Capacity & 0.46 & 0.51 & 0.44 & 0.43 & 0.30 \\
\hline
\end{tabular}

\section{Model Validation}

In the case of the ordered probit model, its goodness-of-fit can be assessed by employing the $\rho^{2}$ measure. Commonly defined as $1-(\mathrm{L}(\beta) / \mathrm{L}(0))$, it measures the fraction of the original log likelihood value explained by the model. In nonlinear models, $\rho^{2}$ is not as intuitive as $R^{2}$ is in regression, but it still gives an indication of the goodness-of-fit of the model. The 0.73 value obtained for this measure is considered as very good in the non-linear model case. To further examine the goodness-of-fit of the ordered probit model, Table 6 presents the 
number of predicted vs. actual buses in each condition rating. The model appears to be giving good predictions for most condition ratings. An exception might be condition rating 2 , where the model predicts correctly 63.3 percent of the buses, but predicts 102 in lower conditions, and 33 in higher. Overall, the model predicts correctly the current condition rating of 88.43 percent of the buses, a number which is quite high.

\begin{tabular}{|c|c|c|c|c|c|c|c|}
\hline \multicolumn{8}{|c|}{$\begin{array}{c}\text { Table } 6 \\
\text { Frequencies of Actual and Predicted Outcomes-Probit Model }\end{array}$} \\
\hline \multirow{3}{*}{$\begin{array}{l}\text { Actual } \\
\text { Condition } \\
\text { Rating }\end{array}$} & \multicolumn{7}{|c|}{ Predicted (number of buses) } \\
\hline & \multirow{2}{*}{$\begin{array}{c}\text { No. } \\
\text { of Buses }\end{array}$} & \multicolumn{5}{|c|}{ Condition Rating } & \multirow{2}{*}{$\begin{array}{c}\text { \% Correctly } \\
\text { Predicted }\end{array}$} \\
\hline & & 0 & 1 & 2 & 3 & 4 & \\
\hline 0 & 194 & 194 & & & & & 100.00 \\
\hline 1 & 425 & 10 & 394 & 21 & & & 92.70 \\
\hline 2 & 368 & 3 & 99 & 233 & 33 & & 63.30 \\
\hline 3 & 156 & & & 40 & 116 & & 74.35 \\
\hline 4 & 639 & & & & & 639 & 100.00 \\
\hline Total: & 1782 & & & & & rall: & 88.43 \\
\hline
\end{tabular}

In the case of the LDF, there is no single measure of model goodness-of-fit (such as $\rho^{2}$ ). To validate this model, the holdout procedure (Rencher 1995) was used. In this procedure, all but one observation is used to compute the classification function, and this function is subsequently used to classify the omitted observation. This process is repeated until each observation is classified by a function based on the other observations. This method was used to estimate the correct prediction rates, while the actual LCF presented in Table 5 is based on the entire set of observations. Table 7 indicates that the model yields predictions that are similar to the ones obtained from the probit model. The overall correct prediction rate of 87.03 percent is again high. ${ }^{4}$ It is worth noting that a quadratic classification function was estimated, thus relaxing the covariance homogeneity 
requirement of the linear function. There was no improvement in the overall correct predictions when this method was used.

\section{Table 7}

Frequencies of Actual and Predicted Outcomes-Linear Discriminant Function

\begin{tabular}{|c|c|c|c|c|c|c|c|}
\hline \multirow{3}{*}{$\begin{array}{l}\text { Actual } \\
\text { Condition } \\
\text { Rating }\end{array}$} & \multicolumn{7}{|c|}{ Predicted (number of buses) } \\
\hline & \multirow{2}{*}{$\begin{array}{c}\text { No. } \\
\text { of Buses }\end{array}$} & \multicolumn{5}{|c|}{ Condition Rating } & \multirow{2}{*}{$\begin{array}{c}\text { \% Correctly } \\
\text { Predicted }\end{array}$} \\
\hline & & 0 & 1 & 2 & 3 & 4 & \\
\hline 0 & 194 & 194 & & & & & 100.00 \\
\hline 1 & 425 & 28 & 371 & 26 & & & 87.29 \\
\hline 2 & 368 & & 108 & 225 & 30 & & 61.10 \\
\hline 3 & 156 & & & 34 & 122 & & 78.20 \\
\hline 4 & 639 & & & & & 639 & 100.00 \\
\hline Total: & 1782 & & & & & Overall: & 87.03 \\
\hline
\end{tabular}

\section{Forecasting}

Commonly, ordered probit models are estimated in the literature, but there is little effort in interpreting the estimated coefficients. To obtain a meaningful insight into the magnitude of the effects of each independent variable, the first derivative of the likelihood function (marginal effects) is needed. The marginal effects (Table 8) show the change in the probability of a bus being in a condition rating due to a one unit increase in some exogenous variable. For example, each additional $10^{5} \mathrm{kms}$ in the life of an OASA bus decreases the probability that it will be in condition rating 4 by 0.0944 and in condition rating 3 by 0.1138 . On the other hand, it increases the probability that it will be in condition rating 2,1 , and 0 by $0.1072,0.086$, and 0.079 , respectively. Based on these marginal effects and the estimated probabilities for each condition rating, the distribution of buses at different condition ratings as a function of bus age for OASA was computed (Figure 2). 


\begin{tabular}{|lrrrrr}
\hline \multicolumn{7}{c|}{ Table 8 } \\
Marginal Effects for Ordered Probit Model \\
\hline Variable & 0 & 1 & 2 & 3 & 4 \\
\hline Age & 0.079 & 0.086 & 0.1072 & -0.1138 & -0.0944 \\
Capacity & 0.007 & 0.001 & -0.0009 & -0.0013 & -0.0011 \\
Mileage & 0.092 & 0.0910 & 0.1001 & -0.1779 & -0.1189 \\
\hline
\end{tabular}

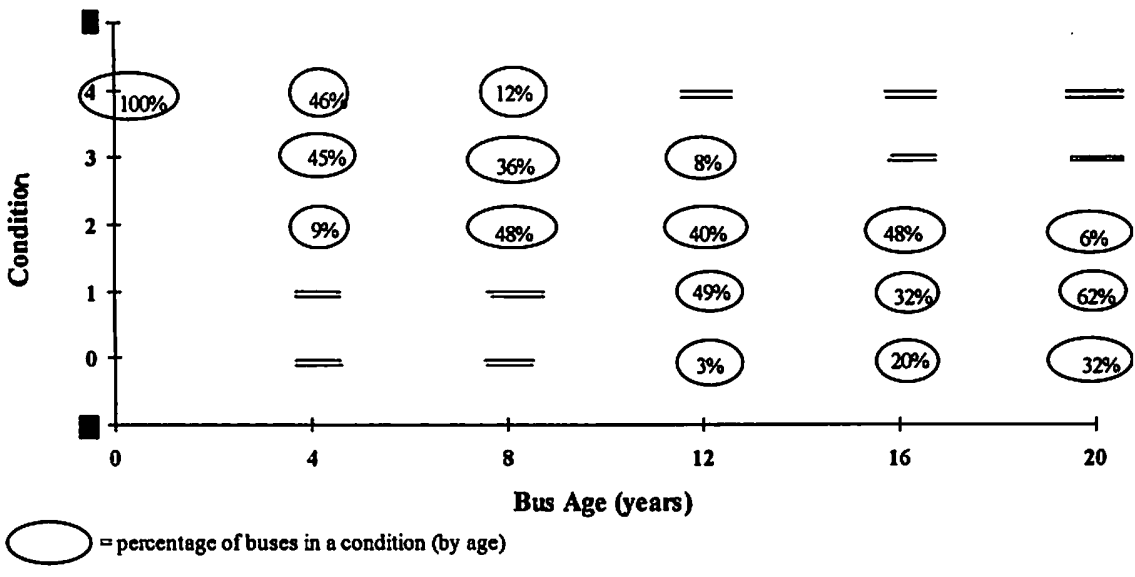

Figure 2. Forecast of the percentage of buses at different condition ratings vs. bus age for OASA

While the ordered probit model and the associated marginal effects can provide good aggregate forecasts of the condition of the entire bus fleet at a point in time, it is very difficult to examine the condition rating of an individual bus. Further, Figure 2 assumes that the other variables (kms and capacity) are at their mean levels. A transit system manager could be interested in the following 
scenario: Can the life of a given bus be prolonged (and by how much) if it is decided to restrict its daily circulation by a certain percentage? This is where the flexibility of the LDF lies. A three-year-old, 40-person bus (seating room) with $95,000 \mathrm{kms}$ is correctly classified as being in condition $4 .{ }^{5}$ The same bus after 15 years in service will be in condition 0 if it has been driven for $910,000 \mathrm{kms}$. If the same bus is driven more prudently $(750,000 \mathrm{kms})$, it will be in condition rating 2 . This type of analysis is very difficult to do using the ordered probit model or any other of the models that have been used to forecast capital asset deterioration. Using this type of process, a large number of "what-if" scenarios can be examined regarding the condition of individual buses under different driving strategies. Hopefully, at a later stage of the development of similar data bases, an explicit measure of the maintenance procedures used on a bus can be incorporated to evaluate the effects of alternate strategies on future condition ratings.

\section{Conclusions}

In this study, two models for examining capital asset deterioration in the transit industry were developed. These models were estimated based on a bus condition data set from Athens, Greece. The first model developed (ordered probit) allows the identification of factors that affect deterioration, as well as the quantification of the magnitude of these (marginal) effects. This way, an understanding of the relative importance of the different explanatory variables on bus condition can be gained. The second model (LDF) provides an easy and rather accurate way in which different mileage (and later on maintenance) scenarios on individual bus deterioration can be examined.

This combination of models provides a way in which to get both aggregate (system level) projections on future bus conditions and disaggregate (individual bus level) projections. Both methodologies used recognize the ordinal nature of condition ratings and link deterioration to a set of relevant explanatory variables such as bus age, mileage, and size. The results can be used easily in a number of practical situations. First, these results can be used to perform bus life-cycle cost analysis, determine optimal timing for bus replacement, and examine the effect of different operational strategies on bus deterioration. Finally, it is worth noting 
that both modeling frameworks, while fairly sophisticated in their theoretical development, are readily available in a large number of commercial computer software packages.

\section{Endnotes}

' In this section, only the essential parts of the ordered probit formulation that might be of interest to the reader are presented. Readers interested in the details of the formulation are encouraged to refer to Greene (1993) for an indepth treatment or Karlaftis and Sinha (1997) for a presentation of the model in the context of rolling stock deterioration.

${ }^{2} \mathrm{Here}$, the very essential parts of discriminant analysis are again presented. For a more thorough analysis, refer to Huberty (1994).

${ }^{3}$ This is a necessary but not sufficient test for multivariate normality. In general, it is recognized that marginal univariate normality is not sufficient for joint normality. Nevertheless, as Stevens (1992) notes, for most practical work one-dimension investigations of normality are ordinarily sufficient. Further, data sets that are normal in lower dimensional representations but nonnormal in higher dimensions are very infrequent in practice (Johnson and Wichern 1992).

${ }^{4}$ In the standard PDF literature, the overall correct predictions are referred to as the "apparent correct classification rate." Frequently, authors use the associated "apparent error rate" (AER), which is $1-0.8703=0.1297$. This AER is considered as very good in the standard PDF literature (Johnson and Wichern 1992).

${ }^{5}$ Classifying this bus in a condition rating using the LDF is very simple. Use the coefficients of Table 5 to obtain the discriminant function score. In this case: $\mathrm{d}_{0}(i)=-234.9, \hat{\mathrm{d}}_{1}(i)=-189.1, \hat{\mathrm{d}}_{2}(i)=-160.6, \hat{\mathrm{d}}_{3}(i)=-110, \mathrm{~d}_{4}(i)=-1.12$. Following the maximum likelihood rule (Eq. (2), this bus can be classified in condition rating 4 .

\section{Acknowledgment}

This research was part of a project for the development of a bus deterioration and forecasting tool for OASA. The author is grateful to the President and staff of OASA for providing the bus condition data base used in this study. 


\section{References}

Federal Transit Administration. 1994. Rail Modernization Study, U.S. Department of Transportation, Washington, D.C.

Galbraith, S. 1996. Development of a Public Transportation Management System in Massachusetts, unpublished Master's Thesis, Department of Civil and Environmental Engineering, University of Massachusetts, Amherst.

Gilbert, E. S. 1968. On Discrimination Using Qualitative Variables, Journal of the American Statistical Association 63: 1399-1412.

Greene, W. H. 1990. Econometric Analysis. New York: MacMillan Publishing Company.

Johnson, R. A., and Wichern, D. W. 1992. Applied Multivariate Statistical Analysis. Englewood Cliffs, N.J.: Prentice Hall.

Huberty, C. J. 1994. Applied Discriminant Analysis. New York: John Wiley and Sons. Karlaftis, M. G., and Sinha, K. C. 1997. A Modeling Approach for Transit Rolling Stock Deterioration Prediction, ASCE Journal of Transportation Engineering, Vol. 123, No. 3: 223-228.

Lachenbruch, P. A. 1975. Discriminant Analysis, Biometrics 35: 69-85.

LIMDEP7. 1995. User's Manual. Bellport, NY: Econometric Software Inc.

Lynch, J., Karlaftis, M. G., Sinha, K. C., and Fricker J. D. 1997. The Indiana Public Transportation Management System, Presented in the $76^{\text {th }}$ TRB meeting, Washington, D.C.

Ludwig, A. 1997. Systems Planning for Capital Asset Management: A Case Study of the New Jersey Public Transportation Facilities and Equipment Management System (PTMS), Presented in the $76^{\text {th }}$ TRB meeting, Washington, D.C.

Madanat, S. M., and Wan Ibrahim, W. H. 1995. Estimation of Infrastructure Transition Probabilities from Condition Rating Data, Journal of Infrastructure Systems, Vol. 1, No.2.

Rencher, A. C. 1995. Methods of Multivariate Analysis. New York: John Wiley and Sons.

Titterington, D. M., Murray, L. S., Speigelhater, D. J., Skene, A. M., Habbema, J. D. F., and Gelpke, D. J. 1981. Comparison of Discrimination Techniques Applied to a Complex Data Set of Head Injured Patients, Journal of the Royal Statistical Society, Series A, 144, 145-175. 
Stevens, J. P. 1992. Applied Multivariate Analysis for the Social Sciences. Hillsdale, NJ: Lawrence Erlbaum.

\section{About the Author}

Matthew G. Karlaftis is in the Department of Engineering in the Greek AirForce Academy. He received his Ph.D. and was a visiting Assistant Professor in the Department of Transportation and Urban Engineering in the School of Civil Engineering at Purdue University in Indiana. His research interests include transportation economics, statistics, air transportation, and ITS. 